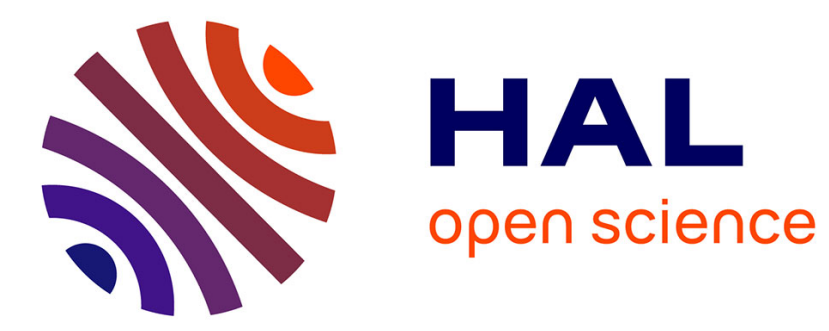

\title{
Les effets des fluctuations du prix du pétrole sur les marchés boursiers dans les pays du Golfe.
}

Mohamed El Hedi Arouri, Christophe Rault

\section{To cite this version:}

Mohamed El Hedi Arouri, Christophe Rault. Les effets des fluctuations du prix du pétrole sur les marchés boursiers dans les pays du Golfe.. 2010. hal-00507825

\section{HAL Id: hal-00507825 \\ https://hal.science/hal-00507825}

Preprint submitted on 1 Aug 2010

HAL is a multi-disciplinary open access archive for the deposit and dissemination of scientific research documents, whether they are published or not. The documents may come from teaching and research institutions in France or abroad, or from public or private research centers.
L'archive ouverte pluridisciplinaire HAL, est destinée au dépôt et à la diffusion de documents scientifiques de niveau recherche, publiés ou non, émanant des établissements d'enseignement et de recherche français ou étrangers, des laboratoires publics ou privés. 


\title{
Les effets des fluctuations du prix du pétrole sur les marchés boursiers dans les pays du Golfe.
}

\author{
Mohamed El Hedi AROURI ${ }^{\$}$ et Christophe RAULT ${ }^{\#}$
}

\begin{abstract}
Résumé. Ce travail utilise les techniques récentes de cointegration en panel et la méthode d'estimation SUR pour tester l'existence d'une relation de long terme entre le prix du pétrole et le cours des actions dans les pays du Conseil de Coopération du Golfe (CCG). Ces pays étant des acteurs majeurs du marché mondial de l'énergie, leurs marchés boursiers devraient être sensibles aux chocs affectant le prix du pétrole. En utilisant une base de données mensuelle couvrant la période allant de janvier 1996 à décembre 2007, notre étude met en évidence, d'une part l'existence d'une relation de cointégration entre le prix du pétrole et le cours des actions dans les pays du CCG, d'autre part, les estimations SUR indiquent que les augmentations du prix du pétrole ont un impact positif sur les rentabilités boursières, sauf en Arabie Saoudite.
\end{abstract}

Mots clés : Marchés boursiers des pays du GC, prix du pétrole, analyse en cointégration sur données de panel.

Classification JEL: G12, F3, Q43.

\begin{abstract}
The aim of this paper is to investigate the existence of long-run relationships between oil prices and stock markets in Gulf Corporation Countries (GCC) using recent bootstrap panel cointegration techniques and Seemingly Unrelated regression (SUR) methods. Since GCC countries are major world energy market players, their stock markets are likely to be susceptible to oil price shocks. Using a monthly dataset covering the period January 1996 to December 2007, our investigation shows that there is evidence for cointegration of oil prices and stock markets in GCC countries, while the SUR results indicate that oil price increases have a positive impact on stock prices, except in Saudi Arabia.
\end{abstract}

Key words: GCC stock markets, oil prices, panel cointegration analysis.

JEL classifications: G12, F3, Q43.

\footnotetext{
\$ Laboratoire d'Économie d'Orléans, CNRS-UMR 6221 (rue de Blois, B.P. 6739, 45067 Orléans Cedex 2), et EDHEC Business School. Email: mohamed.arouri@univ-orleans.fr.

\# Laboratoire d'Économie d'Orléans, CNRS-UMR 6221 (rue de Blois, B.P. 6739, 45067 Orléans Cedex 2), et BEM Bordeaux Management School. Courriel : chrault@ hotmail.com.

Site internet : http://chrault3.free.fr/index.html (auteur correspondant).
}

Nous remercions un rapporteur anonyme pour ses remarques constructives sur une première version de ce texte, ainsi que les participants de la $16^{\text {ème }}$ Conférence Annuelle de l'ERF "Economic Research Forum" intitulée "Chocs, Vulnérabilité et Thérapie", qui s'est tenue au Caire, du 7 au 9 novembre 2009, et plus particulièrement Dr. Rima Turk Ariss, Dr. Faika El-Refaie, et Dr. Osman Suliman. Nous demeurons seuls responsables des éventuelles erreurs et insuffisances de ce travail.

Ce travail a bénéficié d'un financement de l'ERF. Le contenu et les recommandations issues de ce travail ne reflètent pas nécessairement les vues de l'ERF. 


\section{Introduction}

Dans les dernières décennies, de nombreux travaux de recherche ont été consacrés à l'étude des liens entre les prix du pétrole et l'activité économique. Pour l'essentiel, ces travaux ont établi que les chocs des prix du pétrole ont des effets significatifs sur les variables macroéconomiques dans la plupart des pays développés et émergents [Cunado et Perez Garcia de (2005), Balaz et Londarev (2006), Gronwald (2008), Cologni et Manera (2008), Kilian (2008) et Lardic et Mignon (2006, 2008)]. Toutefois, relativement peu d'attention a été accordée à la relation entre les prix du pétrole et les marchés boursiers. En particulier, très peu d'études ont porté sur les marchés boursiers dans les pays émergents. Ces études ont principalement examiné l'interaction à court terme entre les chocs des prix du pétrole et les rentabilités boursières.

En théorie, la valeur d'une action est égale à la somme des flux futurs actualisés. Ces flux actualisés sont affectés par des facteurs économiques qui peuvent être influencés par le prix du pétrole. Ainsi, les fluctuations du prix du pétrole peuvent avoir des effets significatifs sur les cours des actions. La plupart des travaux antérieurs ont étudié cette relation dans le cadre d'un modèle macroéconomique pour des pays importateurs de pétrole. En utilisant des données mensuelles et des tests récents de racine unitaire et de cointégration en panel, ce papier teste l'existence d'une relation de long terme entre le prix du pétrole et le cours des actions dans les pays du Conseil de Coopération du Golfe (CCG).

Étudier les effets de prix du pétrole sur les marchés boursiers du CCG est intéressant pour plusieurs raisons. Tout d'abord, les pays du CCG sont des acteurs principaux du marché mondial du pétrole, leurs marchés boursiers sont donc susceptibles d'être sensibles aux variations des prix du pétrole. Deuxièmement, les marchés du CCG diffèrent des marchés souvent couverts par les études empiriques antérieures par le fait qu'ils sont relativement peu intégrés dans le marché financier mondial et sont extrêmement sensibles aux événements politiques régionaux. Enfin, les marchés du CCG sont très prometteurs pour la diversification internationale de portefeuille. Ainsi, étudier l'influence des chocs des prix du pétrole sur les rentabilités des actifs financiers dans les pays du CCG permet tant aux investisseurs qu'aux autorités de comprendre l'évolution des marchés boursiers en fonction de l'évolution des prix du pétrole.

Le papier pionnier de Jones et Kaul (1996) étudie la réaction à court terme de quatre marchés boursiers développés (Canada, Royaume-Uni, Japon et États-Unis) aux chocs des prix du pétrole. Les auteurs montrent que le prix du pétrole joue un rôle important dans la formation des rentabilités des actifs financiers aux États-Unis et au Canada. Pour les autres pays, l'impact du pétrole était moins évident. En utilisant un modèle VAR, Huang et al. (1996) établissent un lien significatif entre le prix du pétrole et les rentabilités boursières de certaines firmes pétrolières américaines. Cependant, les auteurs ne trouvent pas de relation significative entre le prix du pétrole et des indices de marché américains tel que le S\&P 500. En revanche, Sadorsky (1999) applique un modèle VAR avec des effets GARCH à des données plus récentes et montre que le marché américain réagit significativement aux fluctuations du prix du pétrole. Aloui et Jammazi (2009) montrent l'existence d'une certaine non linéarité dans la relation entre le prix du pétrole et les prix des actifs financiers en France, au Japon et au Royaume-Uni. Qui plus est, en combinant une analyse en ondelettes et des modèles à changement de régime de type markovien (MS-VAR), Jammazi et Aloui (2010) établissent que la réaction des marchés boursiers de ces trois pays aux chocs du prix du pétrole est plutôt asymétrique. Odusami (2009) confirme ces conclusions pour les États-Unis et montre que les chocs inattendus du prix du pétrole ont des effets non linéaires et asymétriques sur les rentabilités boursières. Kilian et Park (2009) mettent en évidence que la réponse des marchés boursiers aux prix du pétrole peut varier sensiblement selon que l'augmentation du prix du pétrole provient d'un choc sur la demande ou d'un choc sur l'offre du pétrole. Enfin, en utilisant la technique de cointégration, Miller et Ratti (2009) montrent l'existence de différents régimes dans la relation à long terme entre le pétrole et le marché boursier dans les pays de l'OCDE au cours des quatre dernières décennies.

Certaines études récentes se sont intéressées au cas des marchés boursiers émergents européens, asiatiques et latino-américains. Les résultats de ces études montrent un lien à court terme significatif entre les variations du prix du pétrole et les rentabilités des marchés boursiers émergents. En utilisant un modèle VAR, Papapetrou (2001) établit l'existence d'une relation significative entre les variations du prix du pétrole et les marchés boursiers en Grèce. Basher et Sadorsky (2006) utilisent un modèle 
multifactoriel d'évaluation des actifs financiers et arrivent à la même conclusion pour d'autres marchés boursiers émergents. Toutefois, très peu d'attention a été accordée aux plus petits marchés émergents, en particulier dans les pays du CCG, où la création des marchés boursiers est relativement récente. En utilisant un modèle VAR, Hamoudeh et Eleisa (2004) montrent qu'il existe une causalité bidirectionnelle entre le marché boursier saoudien et le prix du pétrole. Les résultats suggèrent également que les autres marchés du CCG ne sont pas directement affectés par le prix du pétrole. Zarour (2006) utilise également un modèle VAR pour étudier la relation entre les variations du prix du pétrole et les marchés boursiers du CCG et montre que seuls les marchés saoudien et omanais ont un pouvoir prédictif de l'augmentation du prix du pétrole. Plus récemment, Hamoudeh et Choi (2006) examinent la relation à long terme entre les marchés boursiers du CCG en tenant compte des effets de trois facteurs globaux de risque : le prix du pétrole, un marché américain (S\&P 500) et le taux de bons du Trésor américain. Ils mettent en évidence que le taux de bons du Trésor américain a un effet direct sur ces marchés, tandis que les prix du pétrole et le S\&P 500 ont seulement des effets faibles et indirects. En revanche, Onour (2008) utilisent des données plus récentes et montre qu'à long terme le prix du pétrole affecte significativement le cours des actions dans les pays du CCG. ${ }^{1}$

Comme on peut le constater, les résultats des quelques études consacrés aux effets du pétrole sur les marchés boursiers dans les pays du CCG sont hétérogènes. Ces résultats nous paraissent inattendus car les économies des pays du CCG sont fortement sensibles au prix du pétrole. Le but de cet article est d'approfondir la littérature existante sur le sujet en examinant les liens à long terme entre le prix du pétrole et le cours des actions dans quatre pays du GCC (Bahreïn, Koweït, Oman, et Arabie saoudite), en mobilisant les développements récents de l'économétrie des panels non-stationnaires et la méthode d'estimation SUR ("Seemingly Unrelated Regression"). Plus précisément, nous employons le test de racine en panel développé par Smith et al. (2004), basé sur des techniques de "wild" bootstrap, ainsi que le test de cointégration de la seconde génération proposé par Westerlund et Edgerton (2007), qui tous deux, permettent de tenir compte des éventuelles interdépendances entre les pays considérés. A notre connaissance, une telle analyse n'a jamais été menée pour étudier les liens entre le prix du pétrole et le cours des actions dans les pays du GCC. La mise en œuvre de ces nouvelles méthodes sur un panel de données financières est préférable aux techniques de séries temporelles standard pour remédier au problème aujourd'hui bien connu de la faible puissance des tests de racine unitaire et de cointégration usuels en petits échantillons (comme ce serait le cas ici avec un peu plus de cent observations). L'ajout de la dimension individuelle à la dimension temporelle usuelle présente en effet un intérêt important pour l'analyse des séries non stationnaires puisqu'elle permet d'accroitre la puissance de ces tests [Baltagi et Kao (2000)]. Par ailleurs, et puisque l'influence du prix du pétrole sur le cours des actions requiert d'être examinée pour chaque pays individuellement, une estimation pays par pays est également nécessaire. Dans cette optique, l'approche par la méthode SUR fournit des résultats spécifiques pour chaque pays, ce qui complète ainsi l'analyse en panel.

La suite de cet article s'organise comme suit. La section 2 décrit brièvement les marchés des pays du GCC et le rôle du pétrole. La section 3 présente la base de données et expose les résultats de l'analyse économétrique obtenus avec des tests de racine unitaire et de cointégration en panel de la seconde génération et avec la méthode d'estimation SUR. La section 4 présente les principales implications de nos résultats et la section 5 fournit des éléments de conclusion.

\section{Les marches du CCG et le pétrole}

Le Tableau 1 présente les indicateurs financiers clés des marchés boursiers dans les pays du CCG. Le CCG a été créé en 1981 et comprend six pays: Bahreïn, Oman, Koweït, Qatar, Arabie Saoudite et les Emirats Arabes Unis (EAU). Les pays du CCG ont plusieurs similarités économiques. Ensemble, ils représentent environ $20 \%$ de la production mondiale de pétrole, ils contrôlent $36 \%$ des exportations mondiales de pétrole et ils ont $47 \%$ des réserves mondiales prouvées. Les exportations de pétrole déterminent en grande partie les recettes budgétaires, les dépenses gouvernementales, les résultats des entreprises et la demande globale. Les contributions du pétrole au PIB varient de $22 \%$ (Bahreïn) à

\footnotetext{
${ }^{1}$ Voir aussi Simpson et al. (2004) et Poghosyan et Hesse (2009) qui étudient la relation entre le pétrole et les marches boursiers pour de nombreux pays dont certains pays du CCG. Globalement, leurs résultats sont hétérogènes.
} 
44\% (Arabie Saoudite). En outre, les pays du CCG sont des importateurs de produits manufacturés de pays développés et émergents. Ainsi, les fluctuations des prix du pétrole peuvent influer indirectement sur les marchés du CCG par leur influence sur les prix des importations et l'augmentation des prix du pétrole est souvent révélatrice de tensions inflationnistes dans les économies du CCG. Ainsi, l'étude de l'effet global du prix du pétrole sur les prix des actifs financiers dans les pays du CCG reste essentiellement une question empirique.

L'Arabie saoudite est en tête de la région en termes de capitalisation boursière. Cependant, en pourcentage du PIB, le Qatar est le leader. La capitalisation boursière dépasse le PIB de tous les pays, sauf Oman. Le Koweït a le plus grand nombre de sociétés cotées, suivi d'Oman. Dans l'ensemble, les marchés boursiers du CCG sont limités par plusieurs faiblesses structurelles et réglementaires: un nombre relativement restreint de sociétés cotées, une faible diversification sectorielle, une faible liquidité et plusieurs autres lacunes. Cependant, au cours des dernières années, les marchés boursiers des pays du CCG ont fait l'objet de nombreuses réformes afin de les moderniser et les libéraliser. ${ }^{2}$

Tableau 1- Marchés boursiers du CCG en 2006

\begin{tabular}{lccccc}
\hline Pays & $\begin{array}{c}\text { Nbre de } \\
\text { sociétés cotées* }\end{array}$ & $\begin{array}{c}\text { Capitalisation } \\
\text { (milliards de \$) }\end{array}$ & $\begin{array}{c}\text { Turnover } \\
\text { (milliards de \$) }\end{array}$ & $\begin{array}{c}\text { Capitalisation } \\
\text { en } \\
\text { \% du PIB }\end{array}$ & $\begin{array}{c}\text { Contribution } \\
\text { du pétrole au } \\
\text { PIB }\end{array}$ \\
\hline Bahreïn & 50 & 21.1 & 1.31 & 158 & 22 \\
Koweït & 179 & 143.8 & 59.2 & 190 & 35 \\
Oman & 180 & 12.9 & 2.3 & 40 & 41 \\
Qatar & 36 & 60.9 & 20.5 & 222 & 42 \\
EAU & 102 & 168.7 & 120.4 & 177 & 32 \\
Arabie S. & 86 & 326.3 & 1402.8 & 202 & 44 \\
Sources: Arab Monetary Fund et Emerging Markets Database.
\end{tabular}

Enfin, bien que les pays du CCG aient plusieurs caractéristiques économiques et politiques en commun, ils dépendent du pétrole à des degrés différents, de même, les efforts visant à diversifier et libéraliser l'économie diffèrent d'un pays à l'autre. Le Bahreïn, par exemple, est moins dépendant du pétrole que l'Arabie Saoudite ou le Koweït. D'ailleurs, il est important de souligner l'existence de plusieurs autres différences institutionnelles entre les pays du CCG. Par exemple, contrairement à d'autres marchés boursiers du CCG, le marché saoudien est fortement dominé par des entreprises publiques qui ne sont pas très actives sur le marché boursier. Le marché saoudien est aussi dominé par des institutions financières très dépendantes des pays importateurs du pétrole. En outre, le degré de transparence et la liquidité changent sensiblement d'un pays du CCG à l'autre. Ainsi, la comparaison des marchés boursiers du CCG constitue un sujet intéressant afin de comprendre les effets de ces différences institutionnelles sur la relation entre le pétrole et les actifs financiers.

\section{Etude empirique}

\subsection{Données}

L'objectif de cet article est d'examiner l'existence d'une relation à long terme entre les prix du pétrole et les marchés boursiers dans les pays du CCG. Nous utilisons des données mensuelles fournies par le Fonds Monétaire Arabe (FMA) sur la période Janvier 1996 - Décembre 2007. Il faut noter que les marchés boursiers aux Emirats Arabes Unis et au Qatar ont été creés récemment et n'ont pas participé à la base de données du FMA quand elle a commencé en 2002. Ainsi, les données que nous utilisons ne comporte que quatre des six marchés boursiers du CCG: Bahreïn, Koweït, Oman et l'Arabie Saoudite

\footnotetext{
${ }^{2}$ Pour plus de détails sur les marches boursiers des pays du CCG, le lecteur pourra se référer à Assaf (2003), Creane et al. (2004), Neaime (2005), Al-Khazali et al. (2006), Bley et Chen (2006), Hammoudeh et Choi (2007), Naceur et Ghazouani (2007), Hammoudeh et Li (2008), Arouri et Fouquau (2009) et Hammoudeh et al. (2009).
} 
Pour le pétrole, nous utilisons le prix au comptant mensuel de l'OPEP. Ce prix est pondéré par le volume des exportations et est obtenu à partir de l'Energy Information Administration (EIA). Tous les prix sont exprimés en dollars américains.

Les statistiques descriptives des séries étudiées sont présentées dans le Tableau 2. Le Panel A montre que l'Arabie Saoudite présente la meilleure rentabilité boursière mensuelle suivie par le Koweït et Oman. Le marché boursier omanais présente le niveau de risque le plus élevé suivi par l'Arabie Saoudite et le Koweït. En moyenne, la rentabilité du pétrole est plus élevée que les rentabilités des marchés boursiers du CCG. Le coefficient d'asymétrie (Skewness) est souvent négatif et la statistique du test de Jarque-Bera (JB) rejette fortement l'hypothèse de normalité, à l'exception du Koweït et du pétrole. L'hypothèse d'absence d'effets $\mathrm{ARCH}$ est rejetée pour toutes les séries, sauf pour Oman et l'Arabie Saoudite, alors que l'hypothèse d'absence d'autocorrélations sérielles est rejetée pour le Koweït.

Tableau 2: Statistiques descriptives

Panel A: Statistiques descriptives

\begin{tabular}{lccccc}
\hline \hline & Pétrole & Bahreïn & Koweït & Oman & Arabie S. \\
\hline Moyenne & 1.138 & 0.363 & 0.989 & 0.838 & 1.024 \\
Ecart-type & 8.919 & 1.290 & 4.437 & 8.386 & 7.865 \\
Skewness & -0.398 & -1.071 & -0.059 & 0.157 & -0.200 \\
Kurtosis & 2.875 & 4.663 & 3.741 & 7.905 & 11.713 \\
Jarque-Bera & 3.869 & $22.39 * * *$ & 3.311 & $131.90^{* * *}$ & $377.22^{* * *}$ \\
Q(6) & 2.976 & 1.636 & $13.972^{* *}$ & 4.295 & 5.513 \\
ARCH(6) & 7.594 & 0.217 & 3.151 & $14.966^{* *}$ & $19.821^{* * *}$ \\
\hline \hline
\end{tabular}

Panel B: Corrélations avec le pétrole

\begin{tabular}{ccccc}
\hline & Bahreï & Koweït & Oman & Arabie S. \\
\hline OIL & 0.071 & 0.034 & 0.058 & 0.043 \\
\hline \hline
\end{tabular}

Notes: *, ** et *** indique le rejet de l'hypothèse nulle respectivement à 10\%, 5\% et 1\%. Q(6) et ARCH(6) sont respectivement le test de Ljung-Box d'auto-corrélation d'ordre 6 et le test d'effet ARCH d'ordre 6.

Le Panel B reporte les corrélations entre les variations du prix du pétrole et les rentabilités boursières des marchés des pays du CCG. Ces corrélations sont faibles, mais positives en moyenne. Elles varient de 0,03 (Koweït) à 0,07 (Bahreïn).

\subsection{Tests de racine unitaire en panel}

La littérature concernant les tests de racine unitaire et de cointégration en panel a connu d'importants développements ces dernières années et distinguent entre les tests de la première génération reposant sur l'hypothèse d'indépendance inter-individuelle des individus du panel (à l'exception des effets temporels communs), et les tests de la seconde génération, intégrant diverses formes possibles de dépendances inter-individuelles. Il est aujourd'hui bien connu que les données de panel permettent de travailler avec des échantillons de petite taille dans la dimension temporelle, en considérant un nombre importants d'observations dans la dimension individuelle, ce qui diminue de fait la probabilité d'être confronté à des ruptures structurelles et permet en outre de remédier au problème classique de la faible puissance des tests en petit échantillon. Pour tester l'existence d'interdépendance entre les pays du Golfe, nous avons mis en œuvre le test de Pesaran (2004) et avons calculé le statistique CD. Son hypothèse nulle est l'absence d'interdépendance entre les pays et sous $\mathrm{H}_{0}$ la statistique de test est asymptotiquement distribuée suivant une loi normale. Nos résultats ${ }^{3}$ indiquent que l'hypothèse nulle est toujours rejetée quelque soit le nombre de retards inclus dans la régression auxiliaire augmentée de Dickey-Fuller (jusqu'à cinq retards), au niveau de significativité de cinq pourcents. Ceci confirme que

\footnotetext{
${ }^{3}$ Ces résultats, non reportés ici en raison d'une longueur d'article limitée, sont disponibles sur simple demande.
} 
les pays du CCG sont, comme attendu, très interdépendants, ce qui peut refléter l'existence de règlementations semblables dans plusieurs domaines (au niveau économique, financier, commercial, touristique, législatif, et administratif), une coopération économique, fiscale et politique importante, ainsi qu'une intégration financière sans cesse plus poussée.

Pour déterminer l'ordre d'intégration de nos séries (prix du pétrole, et cours des actions), dans notre panel de pays du CCG, nous utilisons les tests de racine unitaire de la seconde génération proposés par Smith et al. (2004), basés sur une méthode de "Sieve" bootstrap, qui tiennent compte à la fois de la taille de l'échantillon et de la possible interdépendance entre les pays, en générant des valeurs critiques spécifiques aux caractéristiques du panel étudié. Ces tests sont notés ici respectivement $\bar{t}, \overline{L M}, \overline{\max }$, et $\overline{\min }$. Chacun de ces tests suppose, sous l'hypothèse nulle, l'existence d'une racine unitaire pour tous les pays du panel et des racines autorégressives hétérogènes inférieures à l'unité pour chaque pays sous l'hypothèse alternative. Ainsi, en cas de rejet de l'hypothèse nulle, on peut en déduire qu'un pays du panel au moins ne possède pas de racine unitaire et est donc stationnaire ${ }^{4}$. Les résultats reportés dans le Tableau 3, suggèrent que l'hypothèse nulle de racine unitaire ne peut pas être rejetée aux niveaux de significativité usuels pour nos deux séries considérées et cela pour les quatre tests ${ }^{5}$. Nous pouvons donc conclure que toutes nos séries sont intégrées d'ordre un dans notre panel comportant 4 pays du CCG.

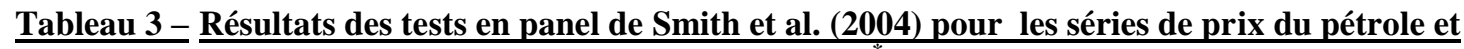
d'indices boursiers" $^{*}$

\begin{tabular}{|c|c|c|c|c|c|c|c|c|}
\hline & \multicolumn{4}{|c|}{ Prix du pétrole } & \multicolumn{4}{|c|}{ Indices boursiers } \\
\hline Test & $\begin{array}{c}\text { Statistique } \\
(\mathrm{a})\end{array}$ & $\begin{array}{c}\text { P-value } \\
\text { Bootstrap } \\
*\end{array}$ & $\begin{array}{c}\text { Statistique } \\
(\mathrm{b})\end{array}$ & $\begin{array}{c}\text { P-value } \\
\text { Bootstrap } \\
*\end{array}$ & $\begin{array}{c}\text { Statistique } \\
(\mathrm{a})\end{array}$ & $\begin{array}{c}\text { P-value } \\
\text { Bootstrap } \\
*\end{array}$ & $\begin{array}{c}\text { Statistique } \\
(\mathrm{b})\end{array}$ & $\begin{array}{c}\text { P-value } \\
\text { Bootstrap } \\
*\end{array}$ \\
\hline $\bar{t}$ & 1.588 & 0.998 & -0.703 & 0.967 & -1.195 & 0.880 & -1.852 & 0.874 \\
\hline$\overline{L M}$ & 2.602 & 0.474 & 0.522 & 0.968 & 1.795 & 0.832 & 2.751 & 0.964 \\
\hline$\overline{\max }$ & 2.602 & 0.259 & 0.517 & 0.940 & -1.684 & 0.321 & -1.741 & 0.784 \\
\hline$\overline{\min }$ & 1.184 & 0.994 & -0.576 & 0.967 & 1.821 & 0.521 & 2.337 & 0.983 \\
\hline
\end{tabular}

Notes: $a$ - Modèle incluant une constante.

$b$-Modèle incluant une constante et un trend linéaire.

* Le rejet de l'hypothèse nulle de non-stationnarité (pour une série donnée) pour l'ensemble des pays du panel, indique la stationnarité de cette série pour au moins l'un des pays du panel. Les p-values bootstrap sont, dans chacun des cas, basées sur 5000 simulations.

\subsection{Tests de cointegration en panel}

Les séries de prix du pétrole et de cours des actions étant intégrées d'ordre 1 , nous implémentons le test de cointégration en panel (de la seconde génération) développé par Westerlund et Edgerton (2007), afin de tester l'existence d'une relation de long terme entre le prix du pétrole le cours des actions des pays du CCG (en accord avec l'équation (1): $\operatorname{Lstock}_{i t}=\alpha+\beta \operatorname{Loil}_{i t}+\varepsilon_{i t}$, où $i$ $(i=1, \ldots, N)$ désigne le pays, et $t(t=1, \ldots, T)$ la période, Lstock la série d'indices boursiers en logarithme, et Loil le prix du pétrole en logarithme). A la différence des tests de cointégration en panel proposés par Pedroni (1999, 2004), et généralisés par Banerjee et Carrion-i-Silvestre (2006), ou

${ }^{4}$ Le test $\bar{t}$ est une version bootstrap du test de panel maintenant bien connu (de la première génération) développé par Im, Pesaran and Shin (IPS, 2003). Les trois autres tests sont simplement des versions modifiées du test d'IPS. Pour davantage de détails sur la construction de ces tests, nous renvoyons le lecteur intéressé à Smith et al. (2004).

${ }^{5}$ Les tests effectués sur les séries en différence première confirment l'hypothèse de stationnarité. En outre, afin d'étudier la robustesse de nos résultats, nous avons également mis en œuvre les tests de racine unitaire de Bai et Ng (2004), de Smith et al.(2004), de Moon et Perron (2004), de Choi (2006), et de Pesaran (2007), qui confirment tous la non-stationnarité stochastique de nos deux séries en accord avec les résultats obtenus avec le test de Smith et al. (2004). Ces résultats sont disponibles sur simple demande. 
celui de Gengenbach et al (2006), ce test présente l'avantage de considérer sous l'hypothèse nulle l'existence d'une relation de cointégration pour l'ensemble des pays composant le panel. Ainsi, en cas de non rejet de cette l'hypothèse, on peut en conclure à l'existence d'une relation de long terme pour l'ensemble des pays du CCG, ce qui est crucial pour en déduire une estimation des paramètres de l'équation (1). En revanche, l'utilisation des autres tests mentionnés précédemment, poserait ici le problème qu'un seul pays pourrait être la cause du rejet de l'hypothèse nulle d'absence d'existence d'une relation de cointégration pour tous les pays du panel, ce qui n'impliquerait pas nécessairement que la relation (1) serait valide pour l'ensemble des pays du CCG. Qui plus est, cette méthodologie ne serait pas alors informative, puisqu'elle ne fournirait aucune information, en cas de rejet de l'hypothèse nulle, concernant le (ou les) pays à l'origine de ce rejet.

Le test développé par Westerlund et Edgerton (2007) est une extension du test du multiplicateur de Lagrange aujourd'hui bien connu de McCoskey et Kao (1998), et permet de prendre en compte les éventuelles corrélations pouvant exister entre les pays du panel considéré. En outre, utilisant une méthode de "Sieve" bootstrap, il présente l'avantage de réduire de manière très importante les distorsions de taille par rapport l'utilisation de la distribution asymptotique (la loi normale), en générant des valeurs critiques spécifiques aux caractéristiques du panel de pays. Il est donc beaucoup plus fiable que les tests de cointégration en panel de la première génération, qui reposent pour la plupart, sur l'utilisation de valeurs critiques issues de la loi normale.

Les résultats de ce test reportés dans le tableau 4 pour un modèle comportant une constante mettent clairement en évidence l'existence d'une relation de cointégration entre le prix du pétrole et le cours des actions pour notre panel de 4 pays du CCG. Ce résultat est cependant basé sur l'utilisation des pvalues asymptotiques qui reposent sur l'hypothèse d'absence de dépendance inter-individuelle entre les pays du CCG, hypothèse qui n'est pas vérifiée ici pour les pays du CCG pour lesquels il existe de fortes dépendances économiques (cf infra).

C'est la raison pour laquelle il est préférable de s'en référer aux p-values bootstrap qui tiennent compte à la fois de la taille finie de l'échantillon, et de la possible dépendance entre les pays. Dans ce cas, les conclusions des tests sont plus immédiates, et si l'on retient un niveau de significativité de $10 \%$, on en conclue à l'existence d'une relation de long terme entre le prix du pétrole et le cours des actions pour notre panel de 4 pays du $\mathrm{CCG}^{6}$. Ce résultat implique en particulier, que dans le long terme, le prix du pétrole et le cours des actions évoluent de manière similaire dans les pays du CCG. Les forces qui agissent sur les marchés du CCG sont en partie celles qui influent sur le prix du pétrole, essentiellement les interventions de l'OPEP, la croissance économique mondiale, ainsi que les tensions sociopolitiques nationales, régionales et internationales.

Tableau 4 - Résultats des tests de cointegration en panel de Westerlund and Edgerton (2007) entre le prix $\underline{\text { du pétrole et les Indices boursiers (selon la spécification de l'équation 1) }}$

\begin{tabular}{lccc}
\hline & Stat-LM & $\begin{array}{c}\mathrm{p} \text {-value } \\
\text { Asymptotique }\end{array}$ & $\begin{array}{c}\mathrm{p} \text {-value } \\
\text { Bootstrap }\end{array}$ \\
\hline Modèle incluant une constante & 10.813 & 0.000 & 0.773 \\
\hline $\begin{array}{l}\text { Notes: Le bootstrap est basé sur 2000 simulations. } \\
\text { a - L'hypothèse nulle du test est l'existence d'une relation de long terme entre le prix du pétrole et les marchés boursiers (cf. } \\
\text { équation 1) pour tous les pays du CCG. }\end{array}$
\end{tabular}

Les coefficients estimés de l'équation (1) sont reportés dans le tableau 5.

\footnotetext{
${ }^{6}$ Il est à noter que nous avons également considéré le cas de cointégration en présence de ruptures structurelles en utilisant le test du Multiplicateur de Lagrange (LM) proposé par Westerlund (2006) qui teste l'hypothèse nulle de cointégration pour tous les pays considérés simultanément, et qui tient compte de l'interdépendance entre les pays considéré en simulant les valeurs critiques par une méthode de bootstrap. Nos Résultats, non reportés ici afin de préserver de l'espace, confirment l'existence d'une relation de cointégration entre le prix du pétrole et le cours des actions pour les pays du CCG, au niveau de significativité de cinq pourcents, tout en ne détectant aucune rupture structurelle.
} 
Tableau 5-Coefficients estimés pour le panel de pays du CCG (Relation moyenne)

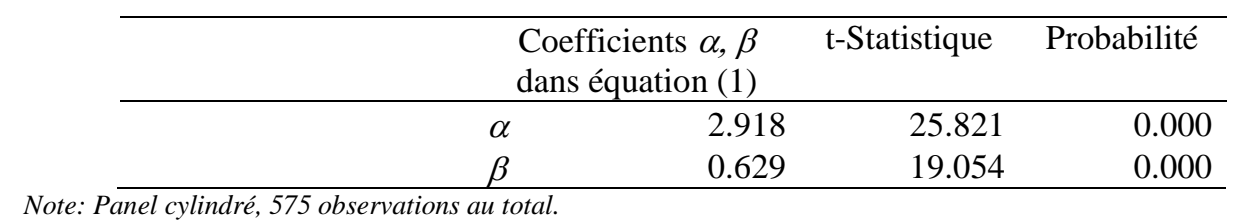

Nos estimations en panel montrent, comme prévu, que le coefficient $\beta$ est significativement positif. L'élasticité du marché boursier au prix du pétrole est inférieure à 1 , mais l'effet d'un choc sur le prix du pétrole est non négligeable : une hausse de $10 \%$ du prix du pétrole entraîne en moyenne une appréciation de $6.29 \%$ des marchés boursiers des pays du CCG.

\subsection{Estimation SUR}

Comme il existe une relation de cointégration pour chacun des pays du GCC dans notre panel, nous estimons à présent l'équation: $\operatorname{Lstock}_{i t}=\alpha_{i}+\beta_{i} \operatorname{Loil}_{i t}+\varepsilon_{i t}, i=1, \ldots, N ; t=1, \ldots, T$ (2), par la méthode SUR développée par Zellner (1962) qui permet de prendre en compte les interdépendances existants entre les pays. Cette manière de procéder nous permet d'obtenir une estimation des coefficients individuels $\beta_{i}$ dans un cadre de panel et d'étudier par conséquent l'influence du prix du pétrole sur le cours des actions de chaque pays pris séparément. Les résultats de ces estimations SUR sont reportés dans le tableau 6.

Tableau 6 - Estimation SUR pour le panel de pays du GCC

\begin{tabular}{|c|c|c|c|c|}
\hline Pays & \multicolumn{2}{|c|}{$\begin{array}{l}\text { Coefficients } \alpha_{i}, \beta_{i} \\
\text { dans l'équation. (2) }\end{array}$} & \multirow{2}{*}{$\begin{array}{r}\text { t-Statistique } \\
27.46\end{array}$} & \multirow{2}{*}{$\begin{array}{r}\text { Probabilité } \\
0.000\end{array}$} \\
\hline Bahreïn & $\alpha_{1}$ & 3.776 & & \\
\hline & $\beta_{1}$ & 0.322 & 8.023 & 0.000 \\
\hline \multirow[t]{2}{*}{ Koweït } & $\alpha_{2}$ & 2.405 & 16.79 & 0.000 \\
\hline & $\beta_{2}$ & 0.827 & 19.76 & 0.000 \\
\hline \multirow[t]{2}{*}{ Oman } & $\alpha_{3}$ & 4.183 & 20.94 & 0.000 \\
\hline & $\beta_{3}$ & 0.195 & 3.346 & 0.000 \\
\hline \multirow[t]{2}{*}{ Arabie Saoudite } & $\alpha_{4}$ & 1.311 & 7.139 & 0.000 \\
\hline & $\beta_{4}$ & -0.384 & -21.83 & 0.000 \\
\hline
\end{tabular}

Note: Panel cylindré, 575 observations au total.

Au niveau pays, la relation entre la variation du prix du pétrole et la rentabilité boursière est positive pour tous les pays du CCG, à l'exception de l'Arabie saoudite. Plusieurs différences économiques et institutionnelles entre le marché saoudien et les autres marchés du CCG pourraient expliquer ce résultat. En effet, le marché boursier saoudien est très concentré et est largement dominé par le secteur financier. Ce dernier est très lié aux marchés financiers des pays occidentaux importateurs du pétrole. Ce manque de diversification peut expliquer la corrélation négative qui a été observée dans les dernières années entre les prix du pétrole et l'indice du marché boursier saoudien. En outre, l'Arabie Saoudite est le plus grand marché du CCG, mais son économie est trop dépendante des pays importateurs de pétrole et souffre plus que les autres pays du CCG de l'inflation importée et des pressions économiques. Enfin, il est important de mentionner que contrairement aux autres marchés 
boursiers du CCG, le marché saoudien est dominé par des entreprises publiques peu actives sur le marché. Ce qui rend le marché peu liquide et peut compromettre les opérations d'arbitrage et de spéculation et donc le fonctionnement normal du marché boursier saoudien.

Pour finir, nous mettons en œuvre des tests de Wald, qui constituent un moyen de mettre en évidence des comportements communs pour des sous-groupes de pays, et de tester l'hypothèse d'homogénéité des $\beta_{i}$ entre les pays. Par exemple, l'on peut chercher à regrouper les pays, selon leur coefficient $\beta_{i}$ estimé (petit ou grand). Les résultats de ces tests sont reportés dans le tableau 7.

$\underline{\text { Tableau } 7 \text { - Test d'homogénéité des } \beta \text { entre les pays }}$

\begin{tabular}{|c|c|c|}
\hline Panel/ groupe de pays & $\begin{array}{c}\text { statistique } \\
\text { du Chi-deux }\end{array}$ & Probabilité \\
\hline$\beta_{i}=1$ pour tous les pays du GCC & 501.06 & 0.0000 \\
\hline$\beta_{i}=\beta$ pour tous les pays du GCC & 238.17 & 0.0000 \\
\hline $\begin{array}{l}\beta_{i}=1 \text { pour tous les pays du GCC } \\
\text { À l'exception de l'Arabie Saoudite }\end{array}$ & 490.647 & 0.0000 \\
\hline $\begin{array}{l}\beta_{i}=\beta \text { pour tous les pays du GCC } \\
\text { À l'exception de l'Arabie Saoudite }\end{array}$ & 107.79 & 0.0000 \\
\hline$\beta_{1}=\beta_{5}$ et $\beta_{2}=\beta_{3}$ & 4.59 & 0.1003 \\
\hline
\end{tabular}

Bien que l'hypothèse nulle d'homogénéité (et de coefficient unitaire) dans la relation de cointégration soit toujours rejetée pour l'ensemble du panel de pays du GCC, cette hypothèse est vérifiée pour certains sous-groupes de pays. Par exemple, l'on observe que l'hypothèse nulle d'homogénéité des $\beta_{i}$, c'est à dire la similarité dans les réponses des marches boursiers des pays du GCC, n'est pas rejetée pour le Bahreïn, le Koweït et Oman considérés simultanément. Néanmoins, malgré l'existence de fortes similarités et de liens économiques forts entre nos 4 pays du GCC, leurs marchés boursiers ont des sensibilités différentes aux variations du prix du pétrole. Enfin, nos résultats suggèrent que les marchés du CCG ont un potentiel diversifiant important et constituent des bons candidats à la diversification internationale de portefeuille.

\section{4- Implications de nos résultats}

En théorie, les fluctuations du prix du pétrole affectent les cours des actions par leurs effets sur les prévisions des bénéfices futurs des entreprises ainsi que sur le taux d'actualisation. Dans la dernière décennie, tant les chercheurs que les praticiens ont tenté de trouver un cadre pratique qui identifie comment les prix du pétrole affectent les cours des actions. Toutefois, ils ne se sont pas parvenus à un consensus général. En utilisant des nouvelles techniques économétriques robustes, nos résultats montrent l'existence de fortes relations à long terme entre le prix du pétrole et quatre marchés boursiers du CCG et que la hausse du prix du pétrole a un effet positif sur le cours des actions dans la plupart des pays du CCG. Nos résultats ont des implications importantes tant pour les chercheurs que pour les acteurs du marché.

Tout d'abord, nos résultats suggèrent qu'une voie intéressante de diversification internationale de portefeuille consisterait à investir simultanément dans les actions des pays importateurs de pétrole (comme la plupart des pays développés) et des pays exportateurs de pétrole (comme les pays du CCG). En effet, nos résultats montrent que les actions de la plupart des pays du CCG ont des sensibilités positives à l'évolution du prix du pétrole. Ces actions peuvent être incluses dans un portefeuille d'actions de pays importateurs de pétrole, généralement avec des sensibilités négatives à l'évolution du prix du pétrole. Une telle stratégie devrait conduire à un risque plus faible, étant donné que le portefeuille constitué est faiblement affecté par les chocs des prix du pétrole.

Deuxièmement, l'existence d'une relation stable à long terme entre les prix du pétrole et les cours boursiers dans les pays du CCG suggère, du point de vue des investissements, que le marché du 
pétrole et les marchés boursiers du CCG sont plutôt intégrés. Ceci implique que les gains attendus d'une stratégie d'investissement en actions des pays du CCG et en pétrole sont décroissants. Ainsi, les investisseurs des pays du CCG devraient chercher à l'étranger pour de nouvelles opportunités d'investissement en vue de constituer des portefeuilles efficients. Toutefois, les investisseurs des pays développés et des autres pays émergents peuvent investir une partie de leur richesse dans les pays du CCG afin de réduire les effets de la hausse des prix du pétrole sur leur rentabilité.

Troisièmement, la relation significative entre le prix du pétrole et les cours des actions implique un certain degré de prévisibilité sur les marchés boursiers du CCG. Sur la base de l'anticipation de l'évolution du marché pétrolier (anticipations des évolutions de l'offre, de la demande et des conditions du marché du pétrole), on peut prévoir la variation future du prix du pétrole et ainsi ses effets sur les prix des actifs financiers dans les marchés du CCG. Ainsi, des stratégies efficaces de spéculation et d'arbitrage peuvent être élaborés à la lumière de nos résultats empiriques.

Enfin, nos résultats montrent que les variations du prix du pétrole affectent de manière significative les marchés boursiers dans les pays du CCG. Les marchés boursiers sont le baromètre de l'activité économique et reflètent fortement le sentiment des consommateurs et des investisseurs. Ainsi, les pays du CCG, en tant qu'acteurs principaux de l'OPEP, doivent accorder une attention particulière à la façon dont leurs décisions au sein de l'OPEP affectent le prix du pétrole et ainsi leurs propres économies et marchés boursiers.

\section{5- Conclusion}

L'objet de ce travail était de tester l'existence d'une relation de long terme entre le prix du pétrole et le cours des actions dans les pays du GCC en mobilisant des tests de racine unitaire et de cointégration en panel de la seconde génération (tenant compte des éventuelles interdépendances existants entre les pays du GCC), et la méthode d'estimation SUR, ce qui à notre connaissance n'avait jamais été réalisé auparavant dans ce contexte. Ces pays étant des acteurs majeurs du marché mondial de l'énergie, leurs marchés boursiers devraient être sensibles aux chocs affectant le prix du pétrole. En utilisant une base de données mensuelle, couvrant la période de janvier 1996 à décembre 2007, notre étude met en évidence l'existence d'une relation de cointégration entre le prix du pétrole et le cours des actions dans les pays du CCG. Nos résultats sont d'intérêt majeur pour les chercheurs, les régulateurs et les acteurs du marché. En particulier, la plupart des pays du CCG en tant que membres de l'OPEP devraient être vigilants aux effets des décisions prises sur le prix du pétrole et donc sur leurs propres économies et marchés boursiers. Pour les investisseurs, la relation significative entre les prix du pétrole et des marchés boursiers implique un certain degré de prévisibilité sur les marchés boursiers du CCG.

Nos résultats suggèrent plusieurs pistes de recherche future. Premièrement, il est raisonnable de penser que le lien à long terme entre le pétrole et les marchés boursiers dans les pays du CCG peut varier d'un secteur économique à l'autre. Une analyse sectorielle de ce lien serait donc intéressante. Deuxièmement, les méthodes économétriques utilisées dans cet article peuvent être utilisées pour examiner les effets d'autres produits énergétiques, comme le gaz naturel, sur l'évolution des marchés boursiers. Enfin, d'autres travaux recherches pourraient examiner les liens de causalité linéaires et non linéaires entre les prix des produits énergétiques, les politiques monétaires et les marchés boursiers dans les pays importateurs et exportateurs de pétrole. 


\section{Références bibliographiques}

- Al-Khazali, O., Darat, A.F. et Saad, M., 2006, Intra-regional integration of the GCC stock markets: The role of market liberalization, Applied Financial Economics 16, 1265-1272.

- Aloui, C. et Jammazi, R., 2009, The effects of crude oil shocks on stock market shifts behaviour: A regime switching approach. Energy Economics 31, 789-799.

- AROURI M (2006), " La prime de risque dans un cadre international: le risque de change estil apprécié?", Finance, 27(1), 131-170.

- $\quad$ AROURI M, FOUQUAU J (2009), "On the Short-Term Influence of Oil Price Changes on Stock Markets in GCC Countries: Linear and Nonlinear Analyses ", Economics Bulletin, vol.29(2), 795-804.

- Assaf, A., 2003, Transmission of stock price movements: The case of GCC stock markets, Review of Middle East Economics and Finance 1, 171-189.

- Bai, J., et Ng, S., 2004. A PANIC Attack on Unit Roots and Cointegration, Econometrica, 72 (4), 127-1177.

- Balaz, P., et Londarev, A., 2006. Oil and its position in the process of globalization of the world economy. Politicka Ekonomie 54 (4), 508-528.

- Baltagi, B. H., et Kao, C., 2000, Nonstationary Panels, Cointegration in Panels and. Dynamic Panels: A Survey, Advances in Econometrics, 15, 7-51.

- Banerjee, A. et Carrion-i-Silvestre, J. 2006. Cointegration in Panel Data with Breaks and Cross-section Dependence, European Central Bank, Working Paper 591, February.

- Bashar, Z., 2006. Wild oil prices, but brave stock markets! The case of Gulf Cooperation Council $(G C C)$ stock markets, Middle East Economic Association Conference, Dubai.

- Basher, S. A. et P. Sadorsky, 2006. Oil Price Risk and Emerging Stock Markets, Global Finance Journal, 17, 224-251.

- Bley, J. et Chen, K.H., 2006, Gulf Cooperation Council (GCC) stock markets: The dawn of a new era, Global Finance Journal 17, 75-91.

- Choi, I., 2006. Combination Unit Root Tests for Cross-sectionally Correlated Panels, in Corbae, D., Durlauf, S. and Hansen, B. (eds), Econometric Theory and Practice: Frontiers of Analysis and

- Cologni, A. et M. Manera, 2008. Oil prices, inflation and interest rates in a structural cointegrated VAR model for the G-7 countries. Energy Economics, 30, 856-88.

- Creane, S., Goyal, R., Mobarak, A.M. et Sab, R., 2004. Financial sector development in the Middle East and North Africa, Working Paper n ${ }^{\circ} 04 / 102$, IMF, Washington, DC.

- Cunado, J., et Perez de Garcia, F., 2005. Oil prices, economic activity and inflation: evidence for some Asian countries. The Quarterly Review of Economics and Finance 45 (1), 65-83.

- Fasano, U., et Iqbal, Z., 2003, GCC Countries: from oil dependence to diversification, International Monetary Fund, Washington D.C.

- Gengenbach C., Palm F.C. et Urbain J-P. (2006). Cointegration Testing in Panels with Common Factors. Oxford Bulletin of Economics and Statistics, 68, 683-719.

- Gronwald, M., 2008. Large oil shocks and the US economy: Infrequent incidents with large effects, Energy Journal, 29, 151-71.

- Hammoudeh, S. et Aleisa, E., 2004. Dynamic relationship among GCC stock markets and NYMEX oil futures, Contemporary Economic Policy, Vol. 22, pp.250-269.

- Hammoudeh, S. et Choi, K., 2007, Characteristics of permanent and transitory returns in oil sensitive emerging stock markets: The case of GCC countries, International Financial Markets, Institutions and Money 17, 231-245.

- Hammoudeh, S. et K. Choi, 2006, Behavior of GCC Stock Markets and Impacts of US Oil and Financial Markets, Research in International Business and Finance, Vol. 20 no. 1, 22-44.

- Hammoudeh, S. et Li, H., 2008, Sudden changes in volatility in emerging markets: The case of Gulf Arab stock markets, International Review of Financial Analysis 17, 47-63. 
- Hammoudeh, S., Yuan, Y. and McAleer, M., 2009. Shocks and volatility spillovers among equity sectors of the Gulf Arab stock markets, Quarterly Review of Economics and Finance 49, 829-842.

- Huang, R. D., Masulis, R. W., et H. R. Stoll, 1996. Energy shocks and financial markets, Journal of Futures Markets, 16, 1-27.

- Im, K., Pesaran, M. et Shin, Y., 2003. Testing for Unit Roots in Heterogeneous Panels, Journal of Econometrics, 115 (1), 53-74.

- Im, K.-S.; Lee, J. et Tieslau, M., 2005. Panel LM unit-root tests with level shifts. Oxford Bulletin of Economics and Statistics, 67 (3), 393-419.

- Jammazi, R. et Aloui, C., 2010, Wavelet decomposition and regime shifts: Assessing the effects of crude oil shocks on stock market returns. Energy Policy 38, 1415-1435.

- Jones, C.M. et Kaul, G., 1996. Oil and the Stock Markets, Journal of Finance, vol. 51, n 2 , pp. 463-491.

- Kilian, L. et Park, C., 2009, The impact of oil price shocks on the US stock market. International Economic Review 50, 1267-1287.

- Kilian, L., 2008. Exogenous Oil Supply Shocks: How Big Are They and How Much Do They Matter for the US Economy?, Review of Economics and Statistics, 90, 216-40.

- Lardic, S. et Mignon, V., 2006, The impact of oil prices on GDP in European countries: An empirical investigation based on asymmetric cointegration, Energy Policy, vol. 34(18), 39103915.

- Lardic, S. et Mignon, V., 2008, Oil prices and economic activity: An asymmetric cointegration approach, Energy Economics, vol. 30(3), 847-855.

- McCoskey, S. et Kao, C., 1998. A Residual-Based Test of the Null of Cointegration in Panel Data, Econometric Reviews, 17 (1), 57-84.

- Miller J.I. et Ratti R.A. 2009, Crude oil and stock markets: Stability, instability, and bubbles. Energy Economics 31, 559-568.

- Moon, H. et Perron, B., 2004. Testing for a Unit Root in Panels with Dynamic Factors, Journal of Econometrics, 122 (1), 8-126.

- Naceur, S.B. et Ghazouani, S., 2007. Stock markets, banks, and economic growth: empirical evidence from MENA region, Research in International Business and Finance, Vol. 21, pp. 297-315.

- Neaime, S., 2005. Financial market integration and macroeconomic volatility in the MENA region: an empirical investigation, Review of Middle East Economics and Finance, Vol. 3, pp. 231-253.

- Odusami B.O., 2009, Crude oil shocks and stock market returns. Applied Financial Economics, 19, 291-303.

- Onour, I.A., 2008, What drives the short-term GCC stock market returns? Empirical evidence from fat-tailed distributions, Afro-Asian Journal of Finance and Accounting 1, 17-25.

- Papapetrou, E., 2001. Oil Price Shocks, Stock Market, Economic Activity and Employment in Greece, Energy Economics, 23, 511-32.

- Pedroni, P., 1999, Critical Values for Cointegrating Tests in Heterogeneous Panels with Multiple Regressors, Oxford Bulletin of Economics and Statistics, 61 (1), 653-670.

- Pedroni, P., 2004, Panel Cointegration; Asymptotic and Finite Sample Properties of Pooled Time Series Tests with an Application to the Purchasing Power Parity Hypothesis, Econometric Theory, 20 (3), 597-625.

- Pesaran, M., 2004. General Diagnostic Tests for Cross Section Dependence in Panels, Cambridge Working Papers in Economics, No 435, University of Cambridge.

- Pesaran, M., 2007, A Simple Panel Unit Root Test in the Presence of Cross Section Dependence, Journal of Applied Econometrics, 22 (2), 265-312.

- Poghosyan, T. et Hesse, H., 2009, Oil prices and bank profitability: Evidence from major oilexporting countries in the Middle East and North Africa, IMF Working Paper No. 09/220.

- Sadorsky, P., 1999. Oil Price Shocks and Stock Market Activity, Energy Economics, vol. 2, pp. 449-469. 
- Sephton, P.S., 1995. Response Surface Estimates of the KPSS Stationarity Test', Economics Letters 47, 255-261.

- Simpson, J., Husain, T. et Evans, J., 2004, The importance of the relationship between OPEC oil prices and GCC banking stock returns, University of Wollongong in Dubai Working Paper No. 21/2004.

- Smith, V., Leybourne, S. et Kim, T.-H., 2004. More Powerful Panel Unit Root Tests with an Application to the Mean Reversion in Real Exchange Rates, Journal of Applied Econometrics 19, 147-170.

- Westerlund, J. et Edgerton, D., 2007, A Panel Bootstrap Cointegration Test, Economics Letters, 97, 185-190, 2007.

- Westerlund, J., 2006, Testing for Panel Cointegration with Multiple Structural Breaks, Oxford Bulletin of Economics and Statistics, 68, 101-132.

- Westerlund, J., 2006. Testing for Panel Cointegration with Multiple Structural Breaks, Oxford Bulletin of Economics and Statistics, 68, 101-132.

- Zarour, B.A., 2006, Wild oil prices, but brave stock markets! The case of GCC stock markets, Operational Research: An International Journal 6, 145-162.

- Zellner, A., 1962. An efficient method of estimating seemingly unrelated regressions and tests of aggregation bias, Journal of the American Statistical Association, 57, 348-368. 